\section{THE CORONIAL AUTOPSY - WHERE ARE WE HEADING?}

Neil Langlois, Helen McKelvie, Philip Beh, Simon Stables, Matthew Lynch, Noel Woodford, David Ranson, John Drayton, Jane Mowll, Chris O'Donnell, Sarah Parsons

This will be a day to consider aspects of the Coronial postmortem examination. The Coronial autopsy, post-mortem examinations performed for the Coroner at his or her direction, are considered to have a special purpose for determination of cause of death and documentation of findings that may have forensic significance for determination of manner of death and use in court if required. Written reference to the autopsy appears in the 12th century, although human dissection for scientific purposes precedes this. Post-mortem examination (although possibly not involving dissection) for investigation of unexplained death dates back to pope Innocentius III in the $13^{\text {th }}$ century. The autopsy as promoted by Morgangi was originally a macroscopic, naked eye assessment with the use of microscopy introduced by Bichat around the end of the 18th century. Rokitansky's method promoted inspection of the whole body and in the late 19th century Virchow published a text describing autopsy technique requiring all the organs to be examined. Have we progressed since then?

Many changes have occurred in the last quarter century. These include the introduction of $\mathrm{x}$-ray computed tomography (CT) scanning and magnetic resonance imaging (MRI). Genetic testing has advanced and is readily available. There has been a substantial decline in the hospital autopsy rate. There have been modifications of Coronial Acts to enable limited examinations and to require the minimum dissection necessary to determine a cause of death. In many States the next of kin are permitted to object to autopsy. Fewer examinations are being ordered in some types of death. Laws and attitudes have changed as a result of retained organs at Alder Hey; organ retention has become arduous and in certain jurisdictions the next of kin can refuse retention of any tissue. It can no longer be assumed that a case referred to the Coroner will have any examination, let alone a dissection of the internal organs. How is it possible to make the best of this situation?

The day will endeavour to consider many of these items as well as addressing perceptions and feelings of the next of kin.

Topics and issues will include:

- Comparison of the legislations

- The Coronial Acts underpin the decisions that are made

- The Acts are not prescriptive, but allow discretion

- Similarities and differences between States

- The Victorian autopsy decision process will be described and discussed

- What is a post-mortem examination?

$\circ$ What are the possibilities? Non-invasive, minimally invasive, invasive?

- Use of histology, microbiology, toxicology, molecular tests

- Should we perform the examination that is appropriate?

- But what is appropriate depends on the information supplied: garbage in = garbage out
- Trading timeliness for completeness, but delay may not be acceptable to family.

- Each case is 'one shot' only - no second chance (particularly if cremated)

- Where death delights to help the living - sometimes

- How often do we achieve an answer?

- Who is asking the questions (family, coroner, clinicians) and what questions do they have?

-What happens when there is no cause of death?

- Using tests

- Don't order a test unless there is a question to answer

- Histology, microbiology, toxicology, neuropathology

- What tests are likely to provide an answer?

$\circ$ How do we get bang for our buck?

- The report

- Who is the report for?

- How do we word it? Plain English or technical terms?

$\circ$ What form(s) could it take?

$\circ$ Timeliness: is there a compromise between speed and quality?

- The Coronial service is not a hospital autopsy service...or should it be?

○ Are hospital pathologists deskilling as the hospital autopsy rate falls?

- Access issue - can clinicians attend a Coronial case and when will they get access to the findings?

- Clinicians' and families' questions may be different to Coronial questions

- Resource issue - can the Coronial system accommodate hospital cases?

- Cultural sensitivities

- New Zealand Maori and other religious/cultural groups

- Retention (or not) of tissue

- Objection to autopsy and what do the next of kin think of the autopsy?

$\circ$ A cultural perspective

$\circ$ What is the point of a medico-legal death investigation system that can be thwarted by the people most likely to have killed the deceased?

o How reliable is the investigation - how do we know it is suicide?

$\circ$ How does one weigh religious objections?

$\circ$ How responsive is the system to cultural needs?

- Organ retention

- Findings of a study involving in-depth interviews with next of kin following autopsy requiring brain retention

- How next of kin was approached

- Responses

- Beliefs regarding usefulness of examination

- Implications

- The meaning of the autopsy and when there is no autopsy for families

- Families are a key client - what do they actually want and what do they expect?

$\circ$ What do families think when there is no autopsy?

- The laparoscopic autopsy

- The minimally invasive autopsy - a video show

○ A viable alternative to a 'limited' autopsy? 
- CT - what is its place?

○ An adjunct or replacement?

$\circ$ Role of angiography - slightly invasive scanning

- The genetic autopsy

- Just because we can, should we?

- How do we communicate the need for family investigation?

- Who does it?

○ What information is released and to who?

- Does it make a difference if testing is additional (e.g., possible familial cancers) or if it goes towards determining cause of death?

- The autopsy for teaching

$\circ$ Who are we teaching?

- An adjunct to learning gross anatomy and pathology

- If full autopsies are not being performed how do our trainees learn to recognise the range of normal?

- The hospital autopsy is declining: How will our future doctors learn what is normal, benign or malignant?

- The Coronial view

- The Coroner is bound by the legislation

- The autopsy is not just for determination of cause of death

- Whether to order an autopsy is a balancing act - A time critical decision often made with limited information

The day will end with a panel discussion answering collated questions from the audience.

Presentations will be brief; some will be factual and didactic, but others may demand audience participation by posing questions and seeking responses using audience response 'clickers'.

Morgangni stated: 'Those who have dissected or inspected many have at least learned to doubt, while those who are ignorant of anatomy and do not take the trouble to attend it are in no doubt at all.' Does this justify advocating examining everything in all cases? Forensic pathologists and Coroners are on the same side: wanting to do what is best. However, assumptions must not be made regarding what is best for the next of kin. Where Coronial Acts require it, the form of the examination should be a considered decision that is evidence based using the available information with due regard to the stated wishes of the family. Forensic pathologists should be able to argue logically (rather than emotively) if an examination of a deceased is required and if a post-mortem examination is to occur, what form it will take and what tests will be required. Is a three-cavity dissection with histological examination, as dictated by history, the ideal investigation in every case? Perspectives and options around this question will be considered; the aim of the day is not to provide answers or policy, but to stimulate you to think about 'The Coronial Autopsy - Where Are We Heading?'

\section{IS PATHOLOGY QA ON THE NOSE?}

\section{$\underline{\text { Paul Bedford }}$}

Victorian Institute of Forensice Medicine, Melbourne, Vic, Australia

Quality assurance programs are needing to bridge the divide between education and proficiency assessments. This issue will be discussed with review of cases from recent forensic QA material.

\section{GENETICS OF THORACIC AORTIC ANEURYSM AND DISSECTION}

Elizabeth Robertson $^{1,2}$, Brett Hambly ${ }^{2}$, Richmond Jeremy ${ }^{1,3}$

${ }^{1}$ Department of Cardiology, Royal Prince Alfred Hospital,

${ }^{2}$ Department of Pathology, and ${ }^{3}$ School of Medicine, University of Sydney, Sydney, Australia

Thoracic aortic aneurysms (TAA) are asymptomatic dilatation of the thoracic aorta that confer a predisposition to dissection, which is often fatal. While the majority are associated with hypertension and atherosclerosis, a significant proportion are due to mutations in proteins within the aortic wall. Some of these mutations result in clinically identifiable syndromes such as Marfan syndrome or Ehlers-Danlos, however some have no discernible physical features at all other than aortic dilatation. Despite the variation in the proteins affected by these genetic mutations, there is a unifying pathological end-point of cystic medial degeneration.

As aortic dilatation is asymptomatic, determining incidence rates amongst those that have non-syndromal TAA (nsTAAD) is difficult. A retrospective cohort analysis of patients in the database at the Sydney Marfan and Aortic Disease Clinic over 20 years, identified that approximately $20 \%$ had nsTAAD, and after family screening, a further 220 affected relatives were identified. This highlights the importance of screening family members of patients who have TAA and dissection, who often first come to attention following sudden death due to dissection. To date, causative genes have been identified in approximately $30 \%$ of patients; therefore, a combination of both genetic diagnosis and clinical screening is warranted.

\section{PATHOLOGISTS AS COMMUNICATORS}

\author{
Philip Beh \\ Department of Pathology, The University of Hong Kong, \\ Hong Kong
}

'The pathologist knows everything but is too late'. This oft repeated punch line in doctor jokes stems from the consistent notion that pathologists deal only with post-mortem examinations. Modern pathology is becoming increasingly diverse with some pathologists no longer performing autopsies and for some such as cytopathologists, never having performed autopsies.Another factor is the 'stereotyping' of personality types and preferences in the choice of medical specialisations; for example, surgeons are 'extroverts' and 'decisive' whereas internists are 'contemplative' and some would say 'indecisive'. Similarly, there is a belief that pathologists are just not 'people persons'. Again, modern pathology is changing rapidly, tests are becoming very complex and interpretations nuanced; it is paramount that pathologists must develop their communicative skills not only with professional colleagues but even more importantly with patients and with the general public.

Medical schools have taken note of the increasing needs of patients and most have included significant exposure to communication skills; perhaps it is time that the specialty acknowledges that this too is a key skill set for pathologists. What good is knowledge and information if it cannot be communicated, or are we as a profession contented with the accusation of irrelevance? 